\title{
Des jardins pour maintenir l'ordre ? Enquête ethnographique dans un quartier populaire strasbourgeois
}

Romane Joly et Vincent Lebrou

\section{(2) OpenEdition Journals}

Édition électronique

URL : https://journals.openedition.org/cdg/7610

DOI : $10.4000 / \mathrm{cdg} .7610$

ISSN : 2107-7266

Éditeur

UMR 245 - CESSMA

Référence électronique

Romane Joly et Vincent Lebrou, « DES JARDINS POUR MAINTENIR L'ORDRE ? ENQUÊTE ETHNOgRAPHIQUE DANS UN QUARTIER POPULAIRE STRASBOURgEOIS», Carnets de géographes [En ligne], 15 | 2021, mis en ligne le 30 avril 2021, consulté le 29 mai 2021. URL : http:// journals.openedition.org/cdg/7610; DOI : https://doi.org/10.4000/cdg.7610

Ce document a été généré automatiquement le 29 mai 2021.

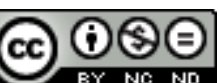

La revue Carnets de géographes est mise à disposition selon les termes de la Licence Creative Commons Attribution - Pas d'Utilisation Commerciale - Pas de Modification 4.0 International. 


\title{
Des jardins pour maintenir l'ordre? Enquête ethnographique dans un quartier populaire strasbourgeois
}

\author{
Romane Joly et Vincent Lebrou
}

\section{Introduction}

Les jardins partagés ${ }^{1}$ se sont multipliés au cours des vingt dernières années dans les villes françaises (Den Hartigh, 2013), au point de s'imposer comme une catégorie à part entière de l'action publique locale ${ }^{2}$. Les discours politiques et médiatiques relatifs à l'aménagement ou la création de ces espaces végétalisés en milieu urbain tendent souvent à les présenter comme le produit d'initiatives spontanées de citoyens désireux de se réapproprier l'espace public ${ }^{3}$. Ils incarneraient l'une des formes contemporaines de l'accomplissement du "droit à la ville ", soit le «droit à la vie urbaine, transformée, renouvelée » (Lefebvre, 2009 [1968]) et viendraient répondre à des besoins de cohésion sociale, de solidarité et de récréation en milieu urbain (Mousselin, Scheromm, 2015). Cette présentation consensuelle, souvent relayée par les collectivités locales, ne résiste néanmoins pas à l'épreuve du terrain. Elle occulte en effet la variété des conditions de création de ces jardins partagés dont cet article entend rendre compte. À partir d'une enquête menée à Strasbourg, nous proposons de revenir sur la création de quatre d'entre eux dans un quartier concerné par la politique de la ville et faisant l'objet d'un plan de rénovation urbaine (encadré 1). Nous montrons ainsi que la fonction sociale de ces nouveaux espaces reste indissociable du contexte dans lequel ils s'inscrivent ainsi que des aspirations des acteurs qui participent, à un moment ou à un autre, à leur conception ou à leur développement. Dans le cas d'étude présenté ici, l'enjeu du maintien de l'ordre finit par reléguer au second plan d'autres formes d'aspiration, par exemple environnementales ou alimentaires ${ }^{4}$, pour le futur de ces jardins.

Encadré 1. Retour sur la création des jardins partagés à Hautepierre 
Hautepierre, où nous avons réalisé notre enquête, est un quartier d'habitat social (celui-ci compte pour $67 \%$ de son parc immobilier) situé à l'Ouest de Strasbourg. Il s'inscrit dans le cadre de la politique de la ville et fait l'objet depuis 2008 d'un large plan de rénovation urbaine. La première renvoie à une série de mesures destinées à apporter des solutions humaines et matérielles aux problèmes sociaux qui se posent dans les quartiers populaires (lutte contre la pauvreté, le chômage ou l'anomie sociale). Le Programme National de Rénovation Urbaine (PNRU), quant à lui, établi par la loi Borloo de 2003, œuvre prioritairement sur le bâti et la morphologie urbaine. Coordonné par l'Agence Nationale pour la Rénovation Urbaine (ANRU) en partenariat avec les collectivités locales, il s'agit notamment d'opérations de démolition d'immeubles vétustes, de résidentialisation ${ }^{5}$ et de réaménagements urbains (Merlin, 2015). A Hautepierre, ce sont principalement ces deux dernières opérations qui ont été mises en œuvre (Vodouhe, 2015).

La question des jardins y est centrale. Faisant d'abord écho au projet initial d'urbanisme de ce quartier inspiré de la « cité jardin » d'Ebenezer Howard (Rudolf, Vodouhe, 2014), il s'agit également d'un projet politique précis dirigé vers ce quartier dans le cadre de la rénovation urbaine. Si un premier jardin partagé avait vu le jour à l'initiative d'habitants dès 2003, la création d'autres jardins est issue des réflexions menées dans le cadre d'un groupe de travail : les Ateliers Territoriaux de Partenaires (ATP). Coordonnés par la municipalité, ils réunissent depuis les années 2000 différents acteurs locaux - associations, bailleur social, services éducatifs - qui s'attachent à résoudre des problèmes d'ordre sécuritaire, jugés caractéristiques de la situation du quartier. L'ATP « réappropriation de l'espace public » est plus particulièrement à l'origine de la création de ces jardins partagés à partir de 2008. Ces derniers sont envisagés dès le départ comme un moyen d'inciter des adultes à réinvestir l'espace public. La création de ces jardins est confiée par la Ville, via un marché public, à une première association strasbourgeoise, EcoConseil, spécialisée dans la sensibilisation aux enjeux environnementaux. Leur développement est progressivement délégué à une association locale, Horizome, garante de leur bon fonctionnement et dont l'une des tâches est d'accompagner les jardiniers à se structurer eux-mêmes en association en vue d'assurer une gestion autonome des jardins. Ces deux organisations ont été sollicitées dans le cadre des ATP, la première en raison de sa spécialisation sur les questions d'agriculture urbaine, la seconde en raison de son implication dans le quartier.

La principale hypothèse de cet article postule que les jardins urbains, et particulièrement les jardins partagés dont il est question ici, sont investis de fonctions différenciées selon leur localisation en ville et les politiques publiques auxquelles ils se rapportent. La création ou l'aménagement de ces nouveaux espaces dans un quartier d'habitat social renvoie davantage à des enjeux de maintien de l'ordre, qui prévalent ainsi, plus ou moins fortement et durablement, sur d'autres fonctions, mentionnée plus haut, généralement attribuées à ces jardins.

Pour qualifier la nature du maintien de l'ordre dont ces nouveaux espaces sont les vecteurs, nous empruntons ici à Michel Foucault la notion de "gouvernementalité » Celle-ci renvoie à un enjeu de " conduite des conduites", soit l'« ensemble des moyens qui permettent d'imposer des normes de comportement à des individus et des 
groupes " (Jeanpierre, 2006). À la différence des interventions policières initialement associées aux politiques de rénovation urbaine de la seconde moitié $\mathrm{du} \mathrm{xx}^{\mathrm{e}}$ siècle, cette gouvernementalité s'articule autour de deux dimensions principales : des " dispositifs » d'encadrement conçus par les autorités, dans notre cas les décideurs publics et responsables associatifs, et des «dispositions entretenues» qui renvoient à la collaboration, consciente ou inconsciente des habitants (Foucault, 1982). Afin de donner à voir la manière dont cette gouvernementalité se concrétise, nous employons le terme foucaldien de " discipline ». Celle-ci désigne les mécanismes de surveillance et de contrôle non-coercitifs qui transparaissent dans l'injonction à l'alignement des conduites selon un modèle prescrit - on parlera aussi ici de "normalisation ». Les jardins partagés relèvent pleinement d'une telle logique dans la mesure où ils sont mobilisés à des fins de normalisation et de pacification, ce que l'on désigne par "maintien de l'ordre ", via le contrôle de l'espace et des conduites. Nous monterons en effet que, comme toute opération de rénovation (Fleury, Froment-Meurice, 2014), l'une des préoccupations dominantes des acteurs impliqués dans le développement de ces jardins consiste à disqualifier certaines conduites perçues comme déviantes au profit d'autres perçues comme plus conformes à l'image qu'ils désirent pour le quartier.

Cette fonction disciplinaire des jardins urbains n'est pas nouvelle (Glatron, Granchamp, 2018) et peut être appréhendée à l'aune du contexte d'émergence de tels espaces. Ainsi, la forme particulière du jardin partagé connaît une double filiation : l'une européenne, comme héritage des "jardins ouvriers" du xIX siècle, l'autre anglo-saxonne comme expression d'un mouvement social des années 1970 revendiquant un «droit à la ville » (Lefebvre, 1974). Si la seconde est bien militante et contestataire, le caractère disciplinaire des jardins urbains émane directement de cette première filiation européenne. Celle-ci est intimement liée aux «jardins ouvriers » créés à la fin du XIX ${ }^{e}$ siècle à l'initiative du patronat et des pouvoirs publics et destinés aux travailleurs. Florence Weber (1998) voit dans ces jardins une appréhension paternaliste des problématiques sociales: il s'agit de s'assurer de la santé physique des travailleurs, mais aussi de moraliser leurs conduites en les recentrant sur la sphère domestique, les tenant à distance de la sphère politique. Le jardin ouvrier était ainsi un instrument d'« éducation » et de contrôle des populations ouvrières dont le jardin partagé, comme le montre notre enquête, peut être le prolongement ${ }^{7}$. Pour autant, cet héritage disciplinaire est régulièrement invisibilisé. Ceci tient d'une part au cadrage politique et médiatique $^{8}$ qui présente ces derniers à l'aune de la filiation anglo-saxonne des "community gardens", comme instrument de réactivation de la citoyenneté (Baudry, 2011)', et d'autre part à leur captation par des populations urbaines privilégiées moins concernées par cet impératif de disciplinarisation des conduites. Cette approche pacifiée des jardins urbains ne fait pas l'unanimité pour autant. Plusieurs auteurs critiques (notamment McClintok, 2014 ; Tornaghi, 2014 ; Horst, McClintock, Hoey, 2017 ; Paddeu, 2017) associent la multiplication récente de ces espaces dans les villes du "Nord", notamment sous un volet social et alimentaire, à des problématiques structurelles liées à la gouvernance urbaine néolibérale. Dans des villes nordaméricaines, l'agriculture urbaine est notamment appréhendée dans un contexte de retrait de l'État où, tout en véhiculant un discours de responsabilisation des populations fragilisée, il s'agit de déléguer la résolution des problèmes sociaux et la sécurité alimentaire aux organisations associatives et bénévoles ainsi qu'aux communautés et individus. D'autres travaux européens voient se dessiner dans les dispositifs de jardinage urbain une figure municipale prescriptrice plutôt que 
pourvoyeuse de services : cela se traduit à Berlin ou à Vernier par l'enrôlement à titre gracieux des habitants dans l'entretien de l'espace public (Rosol, 2012 ; Ernwein, 2017) ou encore par la marchandisation au plus offrant d'espaces urbains vacants à Paris (Demailly, Darly, 2017).

Notre contribution prolonge ces approches critiques, au sens où elle interroge les rapports de pouvoir tels qu'ils se donnent à voir dans la régulation de ces nouveaux espaces. Elle entend pour autant se démarquer des travaux mentionnés plus haut en ce qu'il s'agit d'appréhender, dans un contexte français, les formes que prend l'intervention institutionnelle en matière de jardins urbains, lorsqu'elle est dirigée vers les habitants des quartiers d'habitat social. Notons que Marion Ernwein (2019), s'intéressant à la création de "plantages $~^{10}$ localisés dans des quartiers populaires genevois, y décèle une volonté municipale de transformer les modalités d'investissement de l'espace urbain par les classes populaires. Alors que ces populations investissent traditionnellement des espaces jardinés péri-urbains privatisés où règne l'entre-soi familial, le plantage, au contraire, enjoint les habitants à se recentrer sur la vie de quartier et à participer activement au fonctionnement collectif du jardin, ce qui revient à "transformer les valeurs et normes qui régissent les espaces populaires " (Ibid. : 114). Nous proposons de prolonger ces réflexions sur les injonctions normatives associées à la création de ces espaces, en nous intéressant, dans un tout autre contexte, au caractère disciplinaire que peuvent revêtir les jardins partagés. Celui-ci apparait dès lors que l'on réarticule ces espaces d'une part aux politiques de rénovation urbaine ${ }^{11}$, elles-mêmes largement marquées par des enjeux de maintien de l'ordre (Gosselin, 2015), d'autre part aux profils, contraintes et préoccupations des acteurs impliqués dans leur régulation.

À partir de la question des jardins partagés, nos réflexions renvoient donc directement à la dimension sécuritaire de la rénovation urbaine et aux modalités de sa concrétisation. Renaud Epstein (2015) rappelle que nombreux sont ceux qui ont vu, très tôt, dans les politiques de rénovation urbaine l'apparition d'un urbanisme contrerévolutionnaire ${ }^{12}$. Pour d'autres, les objectifs de ces politiques, notamment par le biais du PNRU, consisteraient à contrecarrer les révoltes qui embrasent sporadiquement les quartiers populaires et donc à faciliter le déploiement des forces de l'ordre contre la délinquance par l'aménagement urbain (Garnier, 2002). Ici, nous entendons davantage appréhender la question sécuritaire par le biais d'enjeux de tranquillité publique (Carrel, 2013). La création de jardins partagés s'inscrirait dans la continuité d'une démarche de "prévention situationnelle $~^{13}$ d'après laquelle l'environnement est un facteur déterminant au passage à l'acte de comportements déviants (Gosselin, 2015), que l'aménagement de l'espace permettrait d'éviter. Dans cette lignée, les jardins partagés sont ainsi investis d'une fonction de pacification de l'espace public qui passe notamment par la facilitation de la sociabilité urbaine dans des lieux dédiés. En cela, ils illustrent cette tendance à la spatialisation des problèmes sociaux (Poupeau, Tissot, 2005) qui postule que la réactivation du lien social doit permettre de résoudre nombre de problématiques qui frappent ces quartiers, notamment celles relevant de la sécurité des habitants.

Dans le cas des jardins que nous étudions ici (encadré 2), l'enjeu politique du maintien de l'ordre se retrouve à deux niveaux principaux. Nous verrons dans un premier temps qu'il trouve à s'incarner à travers la question de l'espace en tant que tel. La volonté commune aux pouvoirs publics et aux habitants de participer à son embellissement traduit une volonté d'instaurer un certain ordre dans le fonctionnement de l'espace 
public. Le maintien de l'ordre se retrouve ensuite dans la redéfinition à l'œuvre, dans le cadre de la création et du développement des jardins, des pratiques et des ressources considérées comme légitimes ou acceptables dans l'espace public.

\section{Encadré 2. Matériau et déroulement de l'enquête}

L'essentiel des données mobilisées dans le cadre de cet article est issu d'une enquête localisée s'inscrivant, au niveau national, dans le cadre du programme de recherche porté par le GIS Démocratie et intitulé Cit'in - Expérimentations démocratiques pour la transition écologique. Celui-ci s'intéresse aux expérimentations citoyennes recouvrant une dimension écologique. Le projet de recherche porté par l'Université de Strasbourg, aborde ces enjeux au travers des questions agricoles et alimentaires, avec le quartier de Hautepierre comme terrain d'étude. Contre l'idée que les comportements écologiques sont l'exclusivité des classes aisées (Comby, 2015), l'hypothèse explorée ici vise à mettre en évidence, dans un quartier populaire, des réseaux d'acteurs qui se sédimentent autour de pratiques de production alimentaire sensibles aux enjeux environnementaux. L'enquête de terrain Cit'in, conduite en 2019, a permis de récolter 16 entretiens avec des acteurs institutionnels (représentant du bailleur social, service de proximité, service des jardins urbains) $(n=3)$, avec des habitants jardiniers $(n=9)$ investis dans des jardins partagés, familiaux, ou situés « en pied d'immeuble » et enfin avec des acteurs associatifs encadrant certains types de jardins - familiaux et pédagogiques parfois eux-mêmes impliqués à titre personnel $(n=4)$. Plusieurs observations dont certaines participantes $(n=3)$ viennent étayer ces données de terrain. Dans le cadre d'une seconde enquête, réalisée pour l'Institut d'Urbanisme et d'Aménagement Régional de l'Université de Strasbourg sur le rapport des classes populaires à l'écologie, des entretiens ont été réalisés avec des habitants jardiniers $(n=3)$, des élus ou agents de différents services de la ville (jardins familiaux, environnement, proximité) $(n=6)$ ou des acteurs associatifs et privés $(n=3)$ investis dans la concrétisation de ces aménagements réalisés en 2016 et 2017 dans le quartier étudié. Ce sont donc au total 28 entretiens, de même que des séances d'observation des réunions relatives au fonctionnement des jardins qui sont mobilisés dans le cadre de cet article.

Aucune des deux recherches évoquées n'avait pour objet la question du maintien de l'ordre. Celle-ci est néanmoins apparue au fur et à mesure que nous avancions dans nos enquêtes respectives, à tel point qu'elle est rapidement devenue un point de convergence entre nos travaux. Les entretiens et observations réalisés ont en effet permis de montrer que l'une des thématiques les plus régulièrement abordées porte sur la définition et la régulation des conduites légitimes au sein de ces nouveaux jardins et, plus largement, dans l'ensemble du quartier. Outre les éléments récoltés dans le cadre de nos enquêtes, le fait qu'ils soient envisagés comme partie prenante de la rénovation urbaine en cours a achevé de nous convaincre de faire du maintien de l'ordre un objet à part entière de notre réflexion commune. 


\section{Les jardins partagés comme instrument de normalisation des espaces}

Cette première partie propose d'appréhender la fonction disciplinaire des jardins partagés d'un point de vue spatial. Ces derniers sont en effets envisagés, par certains services de la Ville, comme un moyen d'agir sur l'esthétique urbaine dans un quartier réputé comme faisant partie des potentiels foyers d'agitation et de révoltes sociales (Bellanger et al., 2018). En introduisant plusieurs jardins au cœur du quartier, il s'agit de concevoir des espaces végétalisés dévolus à la sociabilité, qui participent à véhiculer l'image d'un espace urbain pacifié. Les jardins ont donc d'abord une fonction de présentation où l'esthétique a pour but de restaurer l'image dégradée du quartier. Ils traduisent plus largement une volonté de rétablir un ordre social similaire à celui prôné dans les jardins familiaux marqués par un contrôle régulier des pratiques des jardiniers qui s'y investissent.

\section{La fonction de «présentation » des jardins : embellir et restaurer l'image dégradée du quartier}

Le quartier de Hautepierre pâtit d'une réputation négative. Il est régulièrement considéré comme une zone de relégation par les autres habitants de la ville (Vodouhe, 2015 ; Morovich, 2017), ce qui rejoint des représentations plus généralement relayées dans les discours publics et médiatiques sur la figure du "ghetto " (Wacquant, 2005). Ainsi, un agent de l'antenne locale du bailleur social fait état des « difficultés liées à la concentration de familles, je dirais pauvres enfin en dessous des plafonds de ressources $\aleph^{14}$. De même, un agent du service des jardins familiaux, en charge des jardins urbains sur l'ensemble de la métropole parle de «zones, excusez-moi du terme, parfois de non-droit, où il $\mathrm{y}$ a beaucoup de dégâts, beaucoup de vandalisme $»^{15}$. En raison notamment du taux de pauvreté plus élevé que dans les autres quartiers - 45,9\% contre $22,1 \%$ pour l'ensemble de l'agglomération - et des problèmes de sécurité que l'on y retrouverait, telles que les incivilités ou les trafics entre groupes organisés, Hautepierre fait l'objet d'une attention importante de la part des pouvoirs publics. Ces derniers sont désireux de renouveler son image, notamment en rénovant le quartier. Il s'agit alors d'œuvrer sur l'urbanisme pour améliorer la valeur symbolique de ce quartier (Bellanger et al., 2018), pourtant doté d'une architecture singulière, mais dont le bâti et le mobilier urbain s'est largement dégradé (Rudolf, Vodouhe, 2014). Une telle démarche reprend l'idée selon laquelle les conditions d'habitat comptent parmi les facteurs explicatifs des problématiques sociales que connaissent les grands ensembles (Bidou-Zachariasen, 1997).

Dans un tel contexte, les embellissements que les jardins contribuent à apporter au quartier et les images plus positives qu'ils véhiculent, ont pour but de revaloriser auprès des habitants leur lieu de résidence, que certains vivent comme un stigmate, ce que rappelle cette habitante: "on a mauvaise réputation, je le sais ${ }^{16}$, et ainsi, d'instaurer un climat social pacifié. Postulant que la morphologie d'un lieu prescrit ses usages sociaux (Bellanger et al., 2018), la Ville, par le biais des services concernés ou de son élue en charge des enjeux de "ville nourricière ", considère que l'amélioration des conditions résidentielles a pour corollaire l'apaisement des tensions sociales, par exemple des querelles de voisinages liées à la promiscuité et la vétusté du bâti. En ce 
sens, la création des jardins traduit la concrétisation spatiale de ce que Sylvie Tissot appelle la " politique du lien social » qui veut que l'organisation de l'espace et le lien réactivé entre les individus permettent de résoudre les problèmes sociaux des «quartiers » (Tissot, 2007). Finalement, dans ces espaces « conçus » par les décideurs publics (Lefebvre, 1974), l'aménagement et la normalisation de l'espace ont vocation à participer au maintien de l'ordre.

Par ailleurs, la requalification esthétique du quartier recouvre d'autres enjeux, notamment commerciaux. Ainsi, pour un agent de l'antenne locale du bailleur social, l'embellissement de l'espace urbain par l'entremise des jardins partagés bénéficie à son activité. C'est ainsi un argument en faveur de la commercialisation des logements dont il assure la gestion.

«Le logement est plus facile à louer quand de la fenêtre du logement en question vous voyez des gens qui cultivent leur jardin, ça donne tout de suite une image apaisée du quartier tranquille bucolique. C'est plus agréable à regarder qu'un parking de voitures [...]. C'est un argument de facilitation de la commercialisation de nos logements dans les grands ensembles, ça c'est une chose. Je suis très prosaïque. $»^{17}$

Cet extrait montre bien l'importance accordée à la dimension esthétique des jardins partagés qui participent, aux yeux de cet agent mais aussi des locataires de logements sociaux, à véhiculer une image pacifiée du quartier. Envisagés de surcroît comme des instruments au service de la vente, les jardins partagés font office de "vitrine » du quartier et s'inscrivent de façon plus large dans la promotion par la Ville de ces nouveaux espaces urbains.

En outre, cet enjeu d'embellissement du quartier renvoie à l'intervention d'habitants, engagés dans des pratiques jardinières, et qui souhaitent requalifier leur lieu de résidence. C'est le cas de Kemal, habitant du quartier depuis plusieurs années, qui s'est ainsi approprié un espace jardiné au pied de son immeuble ${ }^{18}$, dont la fonction strictement ornementale traduit sa volonté d'embellir le quartier :

«Ça fait joli, tu t'occupes, et c'est toujours beau. Si tout le quartier ils vont faire la même chose voilà, on va pas... ça serait vraiment trop bien si partout on voyait des fleurs quand on entrait dans un quartier comme ça. $»^{19}$

Cet extrait illustre l'appréciation négative que cet habitant a de l'aspect de son quartier, qu'il dit d'ailleurs frappé par «le bordel» et que le jardin contribue à ordonner :

«Ce serait autrement quand vous passez voir tous les pots partout, tout est fleuri.

C'est pas la même chose quand on passe, on trouve les draps partout, les habits accrochés... voilà après c'est chacun à son idée. $\rrbracket^{20}$

Pour y remédier, cet habitant exhorte à la végétalisation du quartier, projet en cohérence avec la volonté de cet agent de l'antenne locale du bailleur social qui apporte d'ailleurs son soutien, le laissant utiliser l'espace pour cet usage jardinier. Ici, l'embellissement du quartier par le jardinage et la perception d'éléments naturels est mis au service de la requalification d'un environnement urbain déclassé (Manola, 2015). Il participe dans le même temps à véhiculer, chez certains habitants, un idéal d'ordre et de propreté21.

Ainsi, le projet urbain autour des jardins partagés s'attache à leur qualité esthétique, qui, mise au service de la requalification visuelle et symbolique du quartier, a pour objectif la pacification des rapports sociaux ${ }^{22}$. Plus généralement, les aménagements 
dont ces espaces végétalisés font l'objet s'inscrivent plus explicitement aux yeux de certains acteurs impliqués dans un souci de maintien de l'ordre public.

\section{L'ordonnancement de l'espace au service du maintien de l'ordre public}

Ces nouveaux espaces participent également à véhiculer une logique d'alignement des pratiques très largement basée sur le fonctionnement des jardins familiaux (Muramatsu, 2018). Cette démarche est portée par le service dédié à la Ville et se traduit par une remise en cause régulière de leur organisation spatiale et des équipements imaginés par les jardiniers.

Mis en place dans le cadre de la rénovation urbaine du quartier, les jardins de Hautepierre ont fait l'objet d'une attention particulière de la part de la Ville. Alors que différents services municipaux se succèdent dans la coordination de ces jardins, d'abord celui de la prévention urbaine puis celui du « contrat de ville ${ }^{23}$, leur gestion est finalement confiée au service des jardins familiaux. Ce cadrage institutionnel a pour conséquence l'alignement progressif de leurs modalités de fonctionnement sur celles des jardins familiaux ${ }^{24}$, caractérisées par une rationalisation stricte de l'organisation de l'espace (Mousselin, Scheromm, 2015). Par extension, le service désormais en charge de leur suivi fait peser sur les jardins partagés du quartier certaines attentes organisationnelles et d'aménagement qui sont étrangères à leur contexte de création. L'équipement n'est par exemple pas standardisé mais a été créé par les habitants avec le soutien de l'association Horizome. Il s'agit notamment de pavillons, de bancs ou de barrières délimitant les parcelles qui sont faites avec des matériaux de récupération dans le cadre d'après-midi "labo-brico" organisés avec un artiste de la ville. Ces installations sont jugées problématiques et dysfonctionnelles pour un agent des jardins familiaux qui parle d'équipements «construits de bric et de broc $»^{25}$ et qui contreviennent à l'unité souhaitée des espaces jardinés strasbourgeois. Cet agent municipal, qui a fait sa carrière dans différents services et qui occupe ce poste depuis de nombreuses années, appréhende le jardinage urbain de façon fonctionnaliste et gestionnaire ${ }^{26}$. Reconnaissant volontiers être "peut-être de la vieille école mais j'aime bien que tout soit tracé au cordeau $»^{27}$, celui-ci se met en devoir d'équiper les jardins du quartier :

« Pour revenir à Hautepierre, il faut que je réaménage et que je mette tout le monde à niveau [...], je vais leur installer tout ce qu'il faut en clôture, pergola, réseau d'eau etc... $»^{28}$

Cet extrait met en évidence le regard dépréciatif porté sur les installations imaginées par les jardiniers, parfois en collaboration avec les associations présentes dans le quartier. Celles-ci sont en effet considérées comme de piètre qualité et insuffisantes, pour "s'employer vraiment au jardinage ${ }^{29}$, notamment si on les compare aux standards des autres jardins strasbourgeois. Le déclassement opéré par cet agent municipal sur ces installations des habitants corrobore les représentations plus généralement partagées parmi les décideurs publics sur les habitants des quartiers populaires. Ces derniers sont en effet définis par leur «manque et leur déficit » (Tissot, 2007) qu'il s'agirait de pallier. Plus encore, l'extrait plus haut traduit une volonté de d'homogénéisation et de mise en conformité de ces espaces, pourtant appropriés par les habitants, avec le fonctionnement des jardins familiaux. Ceci passe notamment par l'équipement - la construction d'une pergola et de clôtures - ainsi que leur 
organisation parcellaire. Le réaménagement de l'espace, la mise à disposition d'outils spécifiques ainsi que l'ensemble de conduites et de pratiques auxquelles il s'agit de se conformer traduisent les velléités disciplinaires de cet agent qui n'hésite pas à rappeler que « l'on ne fait pas n'importe quoi au jardin $»^{30}$.

Dans cette entreprise d'intégration urbaine, les services de la Ville bénéficient également du relais des associations auxquelles est confié, dans le cadre de la politique de la ville, le développement des jardins en question. Une part importante du travail des acteurs en charge du projet consiste alors à organiser les conditions d'un plus grand respect des espaces communs et prévenir ce qui est considéré comme des actes d'incivilité. Lors des différentes réunions observées, la question des déchets est par exemple régulièrement soulevée car elle représente un obstacle pour certains jardiniers potentiels qui déplorent le manque de respect des espaces partagés. Le travail des associatifs présents s'apparente dès lors à une entreprise incessante de conciliation entre différents enjeux, impératifs et injonctions formulées aussi bien par leurs partenaires institutionnels que par les habitants avec lesquels ils sont amenés à interagir (Bordiec, 2011). Ils doivent à la fois lever les différents obstacles à la venue des jardiniers, tout en rappelant leurs objectifs de départ qui doivent eux-mêmes s'inscrire dans ce que les institutions engagées dans la rénovation du quartier jugent acceptable. Les associations se trouvent en quelque sorte enrôlées dans un processus interactif de définition collective de la fonction des jardins. Si elles n'envisagent pas au premier abord leur action dans une perspective disciplinaire, elles se trouvent de fait engagées dans un processus de contrôle et normalisation des comportements (Bordiec, 2017) induit par une mise en ordre de l'espace conforme à ce que prônent les services de la ville évoqués dans cet article ${ }^{31}$. Celui-ci doit être à nouveau accueillant et en mesure de recevoir tous les habitants qui souhaitent s'y investir, ici dans le cadre d'un jardin partagé.

Le développement des jardins partagés s'inscrit dans une optique de normalisation des espaces urbains au sens où en mettant fin à leur image dégradée, il s'agit d'œuvrer à la réhabilitation symbolique du quartier à l'échelle de la ville. Cela passe ici par un travail collectif d'embellissement des espaces publics et l'alignement de leur fonctionnement sur celui des jardins familiaux. Un tel processus nécessite une implication forte des habitants du quartier. C'est la seconde face de cette disciplinarisation des conduites: au-delà du contenu des prescriptions institutionnelles, les habitants concernés sont enjoints à participer à l'entreprise de disqualification des comportements jugés inadaptés au fonctionnement de ces nouveaux jardins.

\section{Les jardins partagés comme instrument de normalisation des conduites}

L'intervention des services de la Ville - d'abord la Direction de proximité, ensuite le service des jardins familiaux - ne se limite pas à l'action sur la morphologie de l'espace. Elle porte également sur la définition des conduites considérées comme acceptables dans le quartier et que la création de jardins partagés est susceptible de favoriser. Réservant ces espaces à une certaine catégorie d'habitants plutôt qu'à d'autres, jugées déviantes, les services de la Ville successivement impliqués prescrivent et rendent visibles certains types de conduites. Cela est par ailleurs renforcé par l'injonction à 
formaliser les modalités de l'action collective des jardiniers, notamment à travers l'obligation de se constituer en association.

\section{Le jardin comme instrument de relégation des « indésirables »}

Dans le cadre des ATP consacrés à l'espace public, deux objectifs ressortent : il s'agit d'une part de consacrer un espace dévolu aux jardiniers " adultes $»^{32}$ afin de reléguer certaines populations jugées « indésirables » - ici les jeunes - ce qui permet d'autre part de déléguer la sécurisation du quartier aux habitants eux-mêmes en les responsabilisant (encadré 3).

\section{Encadré 3. Les « jeunes » et les jardins}

Isabelle Danic (2016) remarque que dans le cadre de la politique de la ville, le droit à l'espace public est refusé aux populations juvéniles qui sont reléguées vers d'autres lieux de leur quartier (les maisons des jeunes et terrains de sport) ou bien vers la sphère domestique. Ceci rejoint les discours d'une partie importante de nos enquêtés qui souhaitent le plus souvent maintenir les jeunes du quartier à distance. Or, ces derniers ne disparaissent pas pour autant. Au contraire, leur présence est perceptible et souvent présentée sous l'angle de la menace pour le bon fonctionnement des jardins. Les actes de vandalisme et les dégradations sont particulièrement redoutés amenant les jardiniers à composer avec ce jeune public, soit dans la confrontation soit par le biais d'une entente tacite sur les conditions de leur cohabitation. Dans l'un des jardins, la confrontation entre une jardinière et un groupe de jeunes se traduit par des provocations de ces derniers (entrées par effraction dans le jardin, dégradations, jets de détritus) qui affirment avec une intensité croissante leur « droit de présence » (Ripoll, 2006) à mesure que la réponse consiste à clôturer l'espace et à élever les barrières, avec le concours des agents municipaux ${ }^{33}$. Dans un autre jardin au contraire, face à la « menace » latente des jeunes, la tempérance est privilégiée. Comme le souligne Anne : « il faut rester gentils avec les jeunes du quartier parce que sinon ils vont tout nous détruire $\aleph^{34}$. Les jardiniers ferment ainsi les yeux sur les incursions des jeunes dans le jardin ce qui se solde par une cohabitation plus apaisée. Dans les deux cas, on retrouve cette logique de négociation permanente, déléguée ici aux jardiniers du quartier, concernant la définition de ce qu'il est admis de faire ou non dans les jardins.

Un agent de la Direction de la proximité, André, dont le rôle consiste à assurer un travail de coordination entre les différents partenaires de la rénovation urbaine, porte particulièrement ce point de vue. Il s'agit pour lui de contribuer à « remettre l'adulte au cœur de l'espace public, comme référent, en tant qu'éducateur $\aleph^{35}$. Son objectif est de parvenir à une inversion du rapport de force avec ce qui est désigné de façon homogénéisante dans ses propos comme une population de jeunes déviants régulièrement impliqués dans les dégradations du quartier ${ }^{36}$. Ceci rejoint des représentations plus généralement partagées qui imputent les «problèmes des banlieues» (Tissot, 2007) aux populations juvéniles, souvent des hommes racisés, érigées en "nouvelle classe dangereuse » (Chevalier, 1958). Pour André, l'objectif est de mettre fin à la «peur» qu'ils suscitent en donnant les moyens aux adultes 
"d'intervenir par rapport à ce qui se passait dans l'espace public $»^{37}$. Cette préoccupation est centrale dans le lancement de l'ATP. André décrit la période comme "chaude», ce qui nécessite selon lui une réflexion partenariale sur les moyens de remettre de la «discipline» dans la vie du quartier. Dans son discours, le jardin ne constitue alors pas uniquement un espace de loisirs mais peut également être envisagé comme un instrument, parmi d'autres, de relégation de certains habitants aux pratiques considérées comme potentiellement déviantes par l'institution qu'il représente.

Delphine, chargée de mission pour le quartier et préposée à l'organisation des ATP dans la continuité du travail initié par André, reprend cette démarche à son compte, illustrant dans le même temps la permanence d'une représentation partagée :

«Il y a plusieurs objectifs, c'est celui dont je vous parlais au départ, faire en sorte que les espaces publics soient investis pas que par les jeunes mais aussi par les adultes. Faire qu'il y ait une certaine tranquillité dans le quartier. Et aussi faire en sorte qu'il y ait du lien social. Vraiment faire se rencontrer les gens. ${ }^{38}$

L'extrait cité ici confirme la pérennité de la vision véhiculée par la Direction de la proximité des fonctions du jardin. Celui-ci n'est pas mobilisé comme un instrument sécuritaire en tant que tel. Cette dimension s'exprime de manière détournée et prend la forme ici d'un souhait formulé par l'institution de redéfinir la hiérarchie des pratiques acceptables dans l'espace public. Présentée comme une attente légitime et peu discutable, la démarche consistant à rendre l'espace public plus accessible aux adultes se double d'un travail de relégation de populations jeunes et présumées déviantes.

Cette fonction préventive des jardins partagés qui préside à leur création est également partagée par un agent de l'antenne locale de baillage social. Pierre relate les problèmes d'ordre sécuritaire dont le quartier a notamment fait l'objet par le passé avec «des voitures qui flambent en pagaille ${ }^{39}$ et un climat d'insécurité et de peur ressenti par les habitants :

«Qu'est-ce qui pourrait permettre de ramener un peu de calme et de sérénité ? Des adultes référents qui sont là la tête penchée sur la terre, sur leur lopin de terre. [...] L'idée c'était vraiment à la base vous l'avez compris, de marquer le territoire avec des adultes [...]. C'était ça l'idée: s'il y a des adultes dehors, il y aura moins de voitures qui brûlent. $»^{40}$

Cet extrait montre à la fois que le jardin partagé est perçu comme instrument d'éviction d'une population jugée indésirable mais aussi que les jardiniers deviennent garants du maintien de l'ordre qui leur est délégué. La création de ces espaces traduit dès lors une volonté de la Ville de s'en remettre à la participation habitante, par l'exhortation à la sociabilité urbaine et l'investissement de l'espace public, pour y maintenir l'ordre. Elle s'inscrit plus largement dans un contexte de "normalisation et de moralisation des habitants » des «quartiers " entreprise par l'État, faisant de la sécurité « l'affaire de tous » (Le Bart, Lefèbvre, 2002).

A travers cette distinction opérée par plusieurs services de la Ville entre différentes catégories de population susceptibles d'investir l'espace public, c'est la définition des conduites jugées acceptables qui se joue dans la création des jardins partagés. L'accent est alors mis sur le "travail » et "la sociabilité urbaine », ce qui renforce le procédé d'éviction de cette catégorie de population «jeune» qui symbolise, aux yeux de ces acteurs publics, son antithèse. En effet, ces jardiniers besogneux incarnent une conduite opposée à celle de ces «jeunes qui tiennent les murs, qui squattent $»^{41}$, qu'il s'agit d'évincer. Dans ce quartier qui connaît un taux de chômage élevé (de 29,3 \% et de 
$40 \%$ pour les jeunes de moins de 25 ans) mais dans lequel habitent aussi de nombreuses personnes retraitées, le jardinage permet aussi de lutter contre le désœuvrement et de prévenir les comportements déviants qui lui sont associés (incivilités, trafics, vandalisme...) généralement imputées aux populations juvéniles (Danic, 2016).

L'activité de jardinage participe ainsi à la mise en scène d'une morale associée au travail et à la sociabilité urbaine, qu'incarne et relaye la figure du jardinier. En effet, dans ces «espaces conçus » par le politique (Lefebvre, 1974), les jardiniers déploient différentes formes de ressources, notamment collaboratives, telles que le partage de savoirs faires, l'apprentissage du vivre-ensemble, ainsi que la transmission de pratiques agricoles et alimentaires. La fonction disciplinaire - on tente d'imposer ce qu'il convient d'y faire ou non - dont les jardins sont investis à travers la promotion d'une occupation qui conforte l'ordre établi n'est pas sans rappeler les fonctions attribuées aux jardins ouvriers (Weber, 1998). En moralisant les conduites et en recentrant les pratiques dans certains espaces, les jardiniers doivent devenir selon leurs promoteurs le garde-fou de comportements déviants. Le jardin partagé participe alors, dans la continuité de son prédécesseur, à une entreprise d'encadrement des habitants des quartiers populaires.

Les nouveaux jardins partagés implantés dans le quartier n'ont pas uniquement vocation reléguer certaines catégories de population et de comportements. Leur pérennisation passe également par une forme « d'activation douce $»^{42}$ (Astier, 2007) de leurs occupants, incités à se constituer en association afin de parvenir à une gestion en apparence autonome de leur jardin.

\section{Des jardiniers incités à se constituer en association}

Outre la question de la réorganisation de l'espace public, la Ville entend également responsabiliser les habitants du quartier en les incitant à se constituer en association.

Une nouvelle fois, le service des jardins familiaux joue un rôle central. Dans le cadre de ses attributions, il cherche notamment à établir une convention avec les associations gestionnaires des terrains qu'elle met à leur disposition. L'objectif est d'établir des règles communes sur le fonctionnement de ces espaces. Suzanne, membre du service, apparente la signature de ces conventions à un «bail immobilier » comparable à celui que l'on signe quand on entre dans un appartement ou une maison. Sans contrat, « il n'y a pas d'intervention de la Ville par rapport à certaines demandes ${ }^{43}$. La convention comporte une douzaine de pages et rappelle l'ensemble des droits, tels que l'alimentation en eau et l'aménagement de l'espace, ainsi que des obligations contractées par l'association signataire. Parmi ces dernières on retrouve notamment celles consistant à mettre en place un règlement intérieur, à maintenir une ouverture aux habitants du quartier, ou encore à assurer une bonne gestion du terrain qui doit être entretenu. Comme pour tout contrat, les obligations contractées sont susceptibles de donner lieu à des contrôles de la part de la Ville qui peuvent éventuellement aboutir à la résiliation de la convention : «c'est quand même un terrain appartenant à la Ville, en tant que citoyen de Strasbourg, ça me plairait pas de payer des impôts pour quelque chose qui ne fonctionne pas et qui ne suit pas les règlements. C'est évident ${ }^{44}$. Comme le souligne également un agent municipal en charge de l'organisation des jardins, «il faut que ça se fasse dans les règles de l'art $»^{45}$. 
En effet, en cas de non-respect des règlements du jardin et si les habitants ne parviennent pas à se constituer en collectif, le service des jardins familiaux se garde le droit de reprendre à son compte la gestion directe de ces espaces qui jusque-là est dévolue à une association locale. En laissant planer cette menace de la sanction (Astier, 2007), notamment par le biais de l'intervention de contrôleurs qui lui sont directement rattachés, le service des jardins familiaux entend réaffirmer l'autorité municipale sur l'espace et garantir les conduites prescrites. En parlant d'un jardin partagé " problématique » car occupé par des jardiniers non constitués sous forme associative, Oscar, représentant associatif mandaté par la Ville pour l'animation des jardins, s'interroge sur "l'avenir qu'on donne à ce jardin $»^{46}$, c'est-à-dire sa transformation en jardin sous gestion directe de la Ville à défaut de structuration collective de la part des habitants.

Cette sommation a de fortes répercussions chez les jardiniers qui connaissent les risques encourus et se conforment aux modalités d'organisation prescrites par la Ville, ici la constitution en association. Comme l'explique Anne, habitante du quartier et jardinière :

«C'est pour ça qu'on est obligés de faire une association parce que sinon ils [le service des jardins familiaux/la Ville] peuvent nous enlever les jardins. Et nous, on fait l'association pour garder les jardins. $»^{47}$

Cet extrait illustre la force de contrainte de cette menace latente. Elle participe à une mise en conformité des conduites et des modalités organisationnelles qui conditionnent le droit d'accéder à cet espace. Cette force prescriptive dont fait usage la municipalité induit parmi certains jardiniers le développement de postures de défiance vis-à-vis des décideurs publics. Ainsi, alors que surviennent des problèmes de dégradation dans les jardins, une habitante hésite à s'en remettre aux agents municipaux afin d'enrayer la situation :

«Au début j'osais pas trop, j'avais peur qu'on nous prenne le jardin donc je disais pas tout de suite les problèmes qu'on avait et puis c'est quand c'est allé trop loin [le conflit avec les jeunes] qu'on l'a signalé. $\gg^{48}$

Les craintes exprimées et les difficultés rencontrées par ces habitants invitent à questionner le bien-fondé des modalités organisationnelles prescrites par la municipalité. En effet très peu d'entre eux ont auparavant fait l'expérience d'engagements au sein d'associations dont ils ne maitrisent le plus souvent pas les codes (tels que la formation d'un bureau, les rôles dévolus à chacun). Demander aux habitants du quartier d'adopter les mêmes modalités de fonctionnement que celles d'autres jardins urbains strasbourgeois revient alors à tenter d'homogénéiser les conduites - au risque d'exclure les individus qui ne s'y conformeraient pas - et à discipliner les plus acquis au projet. En somme, cette injonction au fonctionnement organisé s'apparente à une invitation à l'autonomisation et à la responsabilisation qui se heurte à l'«individualisme atomisant " (Béroud et al., 2016) que porte en elle la précarité frappant les quartiers comme celui que nous étudions. Cet individualisme rend plus aléatoire la participation à des projets dont le niveau de formalisation croissant augmente les risques d'exclusion des individus les moins dotés en capitaux sociaux et culturels ajustés au fonctionnement collectif exigé par l'institution. 


\section{Conclusion}

Le chercheur qui étudie les «banlieues difficiles» doit se centrer sur « la construction sociale (ou, plus précisément politique) de la réalité livrée à l'intuition et des représentations, notamment journalistiques, bureaucratiques et politiques, de cette réalité » (Bourdieu, 1993). Ces dernières produisent des effets bien réels, notamment dans l'univers politique, par leur propension à structurer les débats sur un sujet donné. Appliqué à notre objet d'étude, cela signifie qu'interroger l'articulation de la dimension spatiale et du maintien de l'ordre dans les quartiers d'habitat social implique de prêter une attention certaine aux intentions exprimées par les principaux acteurs qui s'y investissent. Si la dimension sécuritaire est présente dès le départ, tant la filiation entre jardins partagés et familiaux semble importante, elle ne doit pas être considérée pour autant comme un donné. En effet, les jardins partagés étudiés ici sont envisagés sous l'angle du maintien de l'ordre en raison non seulement du cadre formé par le plan de rénovation urbaine en cours, mais aussi des rapports de force politiques dans lesquels leur développement est inséré. La prégnance acquise au cours des années par le service des jardins familiaux, au détriment par exemple de la Direction de l'environnement, contribue à définir la fonction de ces espaces au prisme des enjeux de maintien de l'ordre.

À rebours de la logique de facilitation de l'intervention que l'on associe aux politiques de rénovation, le maintien de l'ordre par les jardins partagés se joue ici sur un autre registre. Dans le cas étudié, il se définit en effet par trois biais principaux. Il consiste d'abord à rétablir une forme de tranquillité publique dont le quartier se trouverait dénué en raison de nuisances attribuées à certains de ses habitants. Le maintien de l'ordre s'apparente également à une entreprise de «normalisation des comportements des enfants, des adolescents et de leurs parents " (Bordiec, 2011). Les jardins doivent permettre en somme d'apprendre ce qu'il est convenable de faire ou de ne pas faire. Ils participent enfin à l'arrimage de l'idéologie sécuritaire à la morale de l'épanouissement personnel, propice à cet individualisme positif (Béroud et al., 2016) qui veut que les habitants des quartiers populaires se réapproprient la vie de ces espaces urbains, ici en formant des collectifs formalisés et sous contrôle. Au final, l'enjeu du maintien de l'ordre s'inscrit dans cette perspective de spatialisation des problèmes sociaux qui incite à agir sur le bâti et l'apparence d'un quartier tout en reléguant les problématiques économiques et sociales structurelles qui frappent ses habitants.

\section{BIBLIOGRAPHIE}

ASTIER I. (2007), Les Nouvelles règles du social, Paris, Presses universitaires de France.

BAUDRY S. (2011), « Les community gardens de New York City : de la désobéissance civile au développement durable », Revue française d'études américaines, vol. 3, no. 129, pp. 73-86. 
BELLANGER E., COLLET A., DESAGE F., GILBERT P. (2018), « Rénovation urbaine. L'espace comme remède à la question sociale? ", Métropolitiques. En ligne : https://www.metropolitiques.eu/ Renovation-urbaine-L-espace-comme-remede-a-la-question-sociale.html.

BENBOUZID B. (2010), « Urbanisme et prévention situationnelle : le cas de la dispute des professionnels à Lyon », Métropoles, no. 8. En ligne : http://journals.openedition.org/metropoles/ 4391.

BEROUD S., BOUFFARTIGUE P., ECKERT H., MERKLEN D. (2016), En quête des classes populaires, un essai politique, Paris, La Dispute.

BIDOU-ZACHARIASEN C. (1997), « La prise en compte de 'l'effet de territoire' dans l'analyse des quartiers urbains », Revue française de sociologie, vol. 38, no. 1, pp. 97-117.

BORDIEC S. (2011), « Usages de la thématique sécuritaire dans l'éducation populaire. L'offre officielle d'une association de quartier ouverte aux jeunes », Agora Débats/Jeunesses, no. 2, pp. 105-118.

BORDIEC S. (2017), « Diversité limitée. Jeunesses populaires et éducation à la citoyenneté dans les quartiers de la "Politique de la ville" ", Déviance et Société, vol. 41, no. 2, pp. 273-304.

BOURDIEU P., (1993), La misère du monde, Paris, Seuil.

CARREL M. (2013), Faire participer les habitants? Pauvreté, citoyenneté et pouvoir d'agir dans les quartiers populaires, Lyon, ENS Editions.

CEREZUELLE D. (1999), Jardinage et développement social. Du bon usage du jardinage comme outil d'insertion sociale et de prévention de l'exclusion. Guide méthodologique. Paris, Charles Léopold Mayer. En ligne : http://docs.eclm.fr/pdf_livre/105JardinageEtDeveloppementSocial.pdf (consulté le 19/01/21).

CHEVALIER L. (1958), Classes laborieuses et Classes dangereuses à Paris pendant la première moitié du XIXe siècle, Paris, Plon.

CHEVALLIER T. (2018), « Des publics pas si dépolitisés », Politiques de communication, vol. 2, no. 11, pp. 43-72.

COMBY J.-B. (2015), «À propos de la dépossession écologique des classes populaires », Savoir/Agir, vol. 3, no. 33, pp. 23-30.

CORBIN A. (1982), Le Miasme et la jonquille : l'odorat et l'imaginaire social XVIIIe XIXe siècles, Paris, Champs Flammarion.

CORBIN A. (2011), « Préface », in Corteel D., Le Lay S., Les Travailleurs des déchets, Toulouse, Erès, pp. 7-13.

DANIC I. (2016), « Les places des adolescent.e.s en zone urbaine sensible, entre attribution, appropriation et retrait ", Les Annales de la recherche urbaine, $\mathrm{n}^{\circ}$ 111, pp. 78-89.

DEMAILLY K.-E., DARLY S. (2017), « Urban agriculture on the move in Paris: The routes of temporary gardening in the neoliberal city ", ACME: An International Journal for Critical Geographies, vol. 16, no. 2, pp. 332-361. En ligne : https://acme-journal.org/index.php/acme/article/view/ $1384 / 1262$.

DEN HARTIGH C. (2013), « Jardins collectifs urbain : leviers vers la transition », Mouvements, vol. 3, no. 75 , pp. 13-20.

EPSTEIN R. (2013), La Rénovation urbaine, Paris, Presses de Sciences Po. 
EPSTEIN R. (2015), « La démolition contre la révolution. Réactualisation d'un vieux couple », Mouvements, vol. 3, no. 83, pp. 97-104.

ERNWEIN M. (2017), « Urban Agriculture and the Neoliberalisation of What? », ACME - an International Journal for Critical Geographies, vol. 16, no. 2, pp. 249-275. En ligne : https:// www.acme-journal.org/index.php/acme/article/view/1387/1260 (consulté le 19/01/21).

ERNWEIN M. (2019), La nature de la ville néolibérale Une écologie politique du végétal urbain, Grenoble, éditions UGA Université Grenoble Alpes.

FLEURY A., FROMENT-MEURICE M. (2014), « Embellir et dissuader : les politiques d'espaces publics à Paris », in Da Cunha A., Guinand S., (dir.), Qualité urbaine, justice spatiale et projet, Lausanne, Presses polytechniques et universitaires romandes, pp. 67-79.

FOUCAULT M. (1982), « Les techniques de soi », in Foucault M., Dits et écrits III, Paris, Gallimard, coll. « Quarto », pp. 1602-1632.

FOUCAULT M. (1994), Dits et écrits IV, Paris, Gallimard, coll « Quarto ».

FOUILLEUX E., MICHEL L. (dir.) (2020), Quand l'alimentation se fait politique(s), Rennes, Presses Universitaires de Rennes.

GARNIER J-P. (2002), « Un espace indéfendable », Cidades, Communidades e Territorias, no. 5. En ligne : http://1libertaire.free.fr/JPGarnier51.html (consulté le 19/01/21).

GLATRON S., GRANCHAMP L. (2018), The Urban Garden City: Shaping the City with Gardens Through History, New York, Springer International Publishing.

GOSSELIN C. (2015), « La rénovation urbaine et le modèle de 'l'espace défendable' : la montée en puissance des enjeux sécuritaires dans l'aménagement ", Métropolitiques, En ligne : https:// metropolitiques.eu/La-renovation-urbaine-et-le-modele.html (consulté le 19/01/21).

GUITARD E., MILLIOT V. (2015), « Les gestions politiques du propre et du sale en ville », Ethnologie française, vol. 3, no. 153, pp. 405-410.

HAJEK I., HAMMAN P., LEVY J-P. (2015), De la ville durable à la nature en ville, Villeneuve d'Ascq, Presses universitaires du Septentrion.

HORST M., MCCLINTOCK N., HOEY L. (2017), « The Intersection of Planning, Urban Agriculture, and Food Justice: A Review of the Literature ", Journal of the American Planning Association, no. 83, pp. 277-295.

JACOB L., DESAGE F. (2015), « Les aménagements de la participation : design, innovation et controverses socio-spatiales », Lien social et politiques, vol. 1, no.73, pp. 3-12.

JEANPIERRE L. (2006), « Une sociologie foucaldienne du néolibéralisme est-elle possible? », Sociologie et sociétés, vol. 38, no. 2, p. 87-111.

LE BART C., LEFEBVRE R. (2002), « Introduction. Une nouvelle grandeur politique ? », in Le Bart C., Lefèbvre R., La proximité en politique. Usages, rhétorique, pratiques, Rennes, Presses universitaires de Rennes, pp. 11-30.

LEFEBVRE H., HESS R., DEULCEUX S., WEIGAND G. (2009 [1968]), Le droit à la ville, 3e édition, Paris, Economica.

LEFEBVRE H. (1974), « La production de l'espace », L’Homme et la société, no. 31-32, pp. 1532.

LELEVRIER C. (2014), « La rénovation urbaine, un re-peuplement des 'grands ensembles' ? », in Desage F., Morel Journel C., Sala Pala V., Le peuplement comme politiques, Rennes, Presses universitaires de Rennes, pp. 175-194. 
MANOLA T. (2015), « Paysages sensoriels des projets urbains 'durables' : une nature urbaine tiraillée, entre approches techno-décoratives et sensibles. Le cas des quartiers Augustenborg, Bo01, WGT », in Hajek I., Hamman P., Lévy J.-P., De la ville durable à la nature en ville, Villeneuve d'Ascq, Presses universitaires du Septentrion, pp. 211234.

MERLIN P. (2015), « Programme national de rénovation urbaine », dans Merlin P., Choay F., Dictionnaire de l'urbanisme et de l'aménagement, Paris, Éditions Presses Universitaires de France, Coll. « Quadrige », pp. 634-636.

MCCLINTOCK N. (2014), « Radical, Reformist, and Garden-variety Neoliberal: Coming to Terms with Urban Agriculture's Contradictions ", Local Environment, vol. 19, no. 2, pp. 147-171. En ligne : https://core.ac.uk/download/pdf/37767697.pdf (consulté le 19/01/21).

MOROVICH B. (2016) « Quel 'droit de cité' ? L'anthropologie en partage dans les quartiers populaires », Revue des anthropologues, vol. 3-4, no. 146-147, pp. 277-296. En ligne : https:// journals.openedition.org/jda/6547.

MOROVICH B. (2017), Miroirs anthropologiques et changement urbain. Qui participe à la transformation des quartiers populaires, Paris, L'Harmattan, coll. « Anthropologie critique ».

MOUSSELIN G., SCHEROMM P. (2015), « Vers une mise en politique des jardins collectifs urbains. Approche comparée de deux trajectoires municipales à Montpellier et à Lisbonne ", ArticuloJournal of Urban Research, vol. 6. En ligne : https://journals.openedition.org/articulo/2685.

MURAMATSU K., (2018), « Urban gardens in Strasbourg: limited sharing places » in Glatron S., Granchamp L., The Urban Garden City: Shaping the City with Gardens Through History, New York, Springer International Publishing, pp. 307335.

PADDEU F. (2017), « Demystifying Urban Agriculture in Detroit », Metropolitics. En ligne $:$ https:// metropolitics.org/Demystifying-urban-agriculture-in-Detroit.html.

POUPEAU F., TISSOT S. (2005), « La spatialisation des problèmes sociaux », Actes de la recherche en sciences sociales, vol. 4, no.159, pp. 4-9.

RIPOLL F. (2006), « Réflexions sur les rapports entre marquage et appropriation de l'espace », in Bulot T., Veschambre V. (dir), Mots, traces, marques : dimension spatiale et linguistique de la mémoire urbaine, Paris, L'Harmattan, pp. 15-36.

ROSOL M. (2012), « Community Volunteering as Neoliberal Strategy? Green Space Production in Berlin ", Antipode, vol. 44, no. 1, pp. 239-257.

RUDOLF F., VODOUHE G. (2014), « Eco-quartier et quartier jardin : des sémantiques différenciées et adaptées aux grands ensembles pour faire advenir la ville durable ? ", in Menozzi M-J., Les jardins dans la ville entre nature et culture, Rennes, Presses Universitaires de Rennes, pp. 43-56.

TORNAGHI C. (2014), « Critical Geography of Urban Agriculture », Progress in Human Geography, vol. 38 , no. 4 , pp. 551-567.

TISSOT S. (2007), L'Etat et les quartiers. Genèse d'une catégorie de l'action publique, Paris, Le Seuil.

VODOUHE G. (2015), Hautepierre, un éco-quartier modèle pour la ville de Strasbourg : utopie ou réalité, Thèse de doctorat en urbanisme, Université de Strasbourg.

WACQUANT L. (2005), « Les deux visages du ghetto. Construire un concept sociologique », Actes de la recherche en sciences sociales, vol. 5, no. 160, pp. 4-21.

WEBER F. (1998), L'honneur des jardiniers. Les potagers dans la France du XXe siècle, Paris, Belin. 
WOHLFAHRT N. (2003), « The Activating Welfare State in Germany: Beyond the Hartz Commission » in Eick V., Mayer M., Sambale J. (dir), "From Welfare to Work": Nonprofit and the Worfare State in Berlin and Los Angeles. Berlin, JFK Institue et FU Berlin, pp. 12-20.

\section{NOTES}

1. Nous entendons par jardin partagé un « jardin construit et cultivé collectivement par les habitants d'un quartier ou d'un village » et dont l'objectif central est la sociabilité urbaine via des activités culturelles et éducatives de proximité (Mousselin, Scheromm, 2015).

2. Au croisement d'enjeux alimentaires (Fouilleux, Michel, 2020) et de nature en ville (Hajek et al., 2015) les jardins partagés font l'objet d'un traitement politique de la part des autorités publiques locales qui se sont dotées d'élus et de services spécifiquement dédiés à leur création ou à leur entretien quotidien.

3. Voir par exemple Cécile Peltier, "Quand les citadins cultivent la ville», Le Monde, 16 avril 2020.

4. De telles aspirations sont portées pour l'essentiel par le service environnement de la Ville, dont il ne sera pas question ici et qui ne joue, au cours des dernières années, qu'un rôle mineur dans la régulation de ces espaces. Cela tient notamment à sa faible implication dans la rénovation du quartier et au rôle central joué par le service dédié aux jardins familiaux qui ne cherche pas à tisser des liens avec d'autres agents potentiellement porteurs de visions alternatives.

5. La résidentialisation est une opération d'aménagement urbain qui consiste à établir une séparation stricte entre l'espace public et l'espèce privé. Au cœur du PNRU, certains auteurs remarquent qu'elle est mobilisée à des fins de « contrôle » et de lutte contre la délinquance (Lelévrier, 2014, Epstein 2013).

6. Nous reprenons ici la définition de Michel Foucault qui vise à interroger la notion de pouvoir en lien avec les modalités de son exercice et des effets qu'il peut engendrer sur le rapport à soi des individus : «j'appelle "gouvernementalité" la rencontre entre les techniques de domination exercées sur les autres et les techniques de soi » (Foucault, 1994).

7. Il est par exemple significatif que dans le cas strasbourgeois, le suivi des jardins partagés au niveau de la Ville ait été confié au service des « jardins familiaux » (hérité des jardins ouvriers) dont nous verrons plus bas qu'il se distingue par des préoccupations fortes concernant le «bon » fonctionnement des espaces.

8. Le discours véhiculé par l'Eurométropole de Strasbourg insiste sur le caractère " participatif» des jardins partagés dans le cadre desquels la Ville s'engage à soutenir «les habitants qui souhaitent s'investir dans la fabrique d'une ville résiliente, plus verte, plus ouverte, plus durable». Site internet de l'Eurométropole de Strasbourg. http://www.strasbourgcapousse.eu/2 consulté le 29/09/20.

9. Cependant, si l'on se penche sur la genèse des jardins collectifs français, on remarque que les premiers sont créés dans les années 1980 à Bordeaux à l'initiative de l'association «Les Jardins d'aujourd'hui» et ne sont pas l'expression d'une revendication citoyenne mais au contraire sont encouragés par les décideurs publics dans le cadre de la politique de la ville (Cérézuelle, 1999). 
10. Il s'agit de la terminologie suisse, utilisée par les collectivités locales du canton genevois, pour désigner les jardins urbains crées à leur initiative et jardinés collectivement par les habitants.

11. Barbara Morovich (2016) note par exemple à propos du quartier dans lequel les jardins étudiés font leur apparition : "il est apparu que "l'enclavement" des habitants de Hautepierre, mis en avant par les chargés de mission de la rénovation urbaine était un prétexte majeur pour justifier des interventions spatiales, notamment des démolitions et une lecture sécuritaire de l'espace ».

12. Par exemple, la critique inaugurale d'Henri Lefebvre dans Le droit à la ville parle d'urbanisme « contre-révolutionnaire » (1974).

13. Ce concept de « prévention situationnelle » est élaboré à la fin des années 1970 en Grande-Bretagne dans un laboratoire de recherche criminologique du Home office (Benbouzid, 2010). Il a été depuis érigé en catégorie politico-institutionnelle justifiant une intervention sur l'espace pour assurer une plus grande sécurité aux habitants d'un quartier.

14. Entretien avec un agent de l'antenne locale du bailleur social, Strasbourg.

15. Entretien avec un agent des jardins familiaux, Strasbourg.

16. Entretien avec une habitante jardinière du quartier, Strasbourg.

17. Entretien avec un agent de l'antenne locale du bailleur social, Strasbourg.

18. Ces jardins « en pied d'immeuble » tendent à se multiplier. Ils se distinguent en apparence de l'objet principal de cet article. Nous avons néanmoins décidé de les intégrer à l'analyse dans la mesure où ils illustrent l'un des modes d'appropriation possible de ce mot d'ordre de végétalisation du quartier porté par la Ville et de l'implication attendue des habitants pour parvenir à sa concrétisation.

19. Entretien avec un habitant jardinier du quartier, Strasbourg.

20. Ibid.

21. Stéphanie Guitard et Virginie Milliot (2015) rappellent qu'en milieu urbain, les tensions autour du propre et du sale sont d'excellents analyseurs permettant de « saisir les négociations quotidiennes de la civilité ». Les impératifs de propreté vont rejoindre ceux de l'ordre public pour légitimer une redistribution des différentes catégories de citadins dans l'espace urbain et un contrôle des classes laborieuses au sein de logements jugés insalubres (Corbin, 1982, 2011). Souvent associé au contrôle des foules, cet impératif de propreté trouve alors également à s'incarner à travers une volonté d'agir, ici sur les pratiques des habitants-jardiniers.

22. Il est ainsi possible de considérer que ces nouveaux aménagements s'inscrivent directement dans la continuité d'un « design social » de plus en plus présent dans les quartiers d'habitat social et qui cherche à rendre un espace dégradé de la ville plus « désirable » (Jacob, Desage, 2015).

23. Il s'agissait d'un dispositif d'accompagnement à la revalorisation de certains territoires urbains œuvrant notamment sur le logement, les services sociaux et l'éducation. Porté de façon conjointe par l'État, les collectivités locales et leurs partenaires, il a été remplacé par le contrat urbain de cohésion sociale puis par la politique de la ville en 2015.

24. Les jardins familiaux (anciennement jardins ouvriers) sont des parcelles de terrain mises à disposition des habitants par les municipalités. Elles sont gérées de façon individuelle contrairement aux jardins partagés qui sont gérés collectivement.

25. Entretien avec un agent du service des jardins familiaux, Strasbourg.

26. Par « fonctionnaliste et gestionnaire » nous entendons le fait que cet agent attribue une fonction - ici vivrière et de mise en ordre - aux jardins urbains laquelle doit guider 
son aménagement. Son approche témoigne également d'une rationalité gestionnaire au sens où les jardins sont pensés en termes d'efficacité et de productivité (Ernwein, 2019). 27. Ibid.

28. Ibid.

29. Ibid.

30. Ibid.

31. Nos observations montrent en effet que lors des réunions de préparation avec les jardiniers, l'un des principaux enjeux est de parvenir à lever les craintes des jardiniers concernant les potentielles dégradations dont les jardins partagés pourraient être le théâtre et l'objet.

32. Entretien avec un agent de l'antenne locale du bailleur social, Strasbourg.

33. Entretien avec une habitante jardinière, Strasbourg.

34. Entretien avec une habitante jardinière, Strasbourg.

35. Entretien avec un agent de la Direction de proximité, Strasbourg.

36. Barbara Morovich (2016) rappelle que cette distinction opérée au sein de la population se retrouve dans les discours des acteurs du projet de rénovation urbaine et dans les plaquettes liées à la concertation: "ce terme ("forces vives") définit des habitants et des associations appelés par leurs compétences, à participer de manière active et implique dans les processus de concertation, mais indique également un classement entre ces "forces vives" (dont une partie seulement réside sur le quartier) et "d'autres" qui ne sont pas clairement nommées, mais qui sont définies à plusieurs reprises dans les réunions (jeunes "déviants", femmes "soumises", "cas sociaux", etc.) ».

37. Ibid.

38. Entretien avec une chargée de mission pour le quartier, Strasbourg.

39. Entretien avec un agent de l'antenne locale du bailleur social, Strasbourg.

40. Ibid.

41. Ibid.

42. Ceci renvoie à une logique à l'œuvre dans les dispositifs sociaux consistant à exhorter les populations démunies à participer à l'amélioration de leurs conditions de vie et à se responsabiliser plutôt qu'à leur offrir un service social. En Allemagne, le terme d'activation (Aktivierung) est explicitement pris pour mot d'ordre dans les politiques sociales (Wohlfahrt, 2003 ; Chevallier, 2018).

43. Entretien avec un agent du service des jardins familiaux, Strasbourg.

44. Ibid.

45. Entretien avec un agent du service des jardins familiaux, Strasbourg.

46. Entretien avec un membre associatif de EcoConseil, Strasbourg.

47. Entretien avec une habitante jardinière, habitante, Strasbourg.

48. Entretien avec une habitante jardinière, Strasbourg. 


\section{RÉSUMÉS}

La littérature abonde sur les jardins partagés qui, depuis une dizaine d'années, verdissent nombre d'espaces urbains à travers le monde. Rejoignant des enjeux alimentaires et de durabilité urbaine, ces initiatives citoyennes sont présentées dans le discours politique et médiatique en vigueur en France comme l'accomplissement d'un « doit à la ville » par des habitants désireux de se réapproprier l'espace urbain. Pour autant, la fonction attribuée aux jardins partagés apparait moins définitive qu'il n'y parait, dès lors que l'on s'intéresse à la diversité des espaces où ils se déploient ainsi qu'aux personnes impliquées dans leur création et leur fonctionnement. Une enquête ethnographique, menée entre 2016 et 2019 dans un quartier d'habitat social à Strasbourg, met en lumière le caractère disciplinaire conféré aux jardins partagés lorsqu'ils se rattachent à la politique de la ville et s'articulent à un projet de rénovation urbaine. Cet article montre ainsi que ces espaces participent, au sens foucaldien, au gouvernement des conduites des habitants en œuvrant sur trois niveaux principaux. Spatial d'abord, lorsque la végétalisation et l'ordonnancement du quartier sont mis au service de la tranquillité publique. Ensuite, en dédiant certains espaces au jardinage, il s'agit de définir des comportements qui y sont autorisés et de reléguer les populations jugées « déviantes » qui ne s'y conformeraient pas. Enfin, l'exhortation municipale, sous peine de sanction, à formaliser l'action collective des jardiniers, ici en se constituant en association, participe à imposer des comportements dont la dissonance avec les ressources dont disposent certains habitants menace d'exclusion les plus démunis et les moins acquis au projet.

A growing body of literature examines community gardens which, for a decade, are developing in cities across the world. Valued for embracing sustainable and social concerns, political and media discourses in France tend to associate these initiatives with a "right to the city" claimed by urban dwellers. However, this assessment is nuanced as soon as one investigates the spatial and social discrepancies existing between those gardens, that is, the places in cities where they are located and the people involved in their management. An ethnographical research, conducted between 2016 and 2019 in a working-class neighborhood of Strasbourg, reveals the disciplinary mechanisms at play when community gardens are articulated with top-down social policies and urban renewal projects. Using a foucaldian lens, this article demonstrates the governing of people's behaviors through and by these urban spaces. First, the greening and ordering of the neighborhood is primarily about safeguarding public security and order. Second, allocating space for gardening participates in defining which practices and behaviors are allowed or not, and in relegating those accused of deviance. Finally, encouraging - under constraint - gardeners to formalize a community organization consists of imposing certain behaviors and contributes to excluding the most deprived ones.

\section{INDEX}

Thèmes : Carnets de recherches

Mots-clés : jardins partagés, agriculture urbaine, quartier populaire, politique de la ville, gouvernementalité, rénovation urbaine

Keywords : Community Gardens, Urban Agriculture, Working-Class Neighborhood, Politique de la Ville, Governmentality, Urban Renewal 


\section{AUTEURS}

ROMANE JOLY

Doctorante en sociologie, Université de Strasbourg, laboratoire Sociétés, Acteurs, et Gouvernement en Europe (SAGE).

romane.joly[at]etu.unistra.fr

\section{VINCENT LEBROU}

Maître de conférences en science politique, Université de Franche-Comté, Centre de recherches juridiques de l'Université de Franche-Comté (CRJFC). Chercheur associé au laboratoire SAGE de l'Université de Strasbourg.

vincent.lebrou[at]univ-fcomte.fr 